\title{
Molecular evolution in Panagrolaimus nematodes: origins of parthenogenesis, hermaphroditism and the Antarctic species $P$. davidi
}

\author{
Samantha C Lewis ${ }^{1}$, Leslie A Dyal ${ }^{1}$, Caroline F Hilburn ${ }^{1}$, Stephanie Weitz ${ }^{1}$, \\ Wei-Siang Liau ${ }^{2}$, Craig W LaMunyon ${ }^{2}$ and Dee R Denver*1
}

Address: ${ }^{1}$ Department of Zoology and Center for Genome Research and Biocomputing, Oregon State University, Corvallis, Oregon, 97331, USA and ${ }^{2}$ Department of Biological Sciences, California State Polytechnic University, Pomona, California, 91768, USA

Email: Samantha C Lewis - samantha.lewis@usermail.com; Leslie A Dyal - lesliedyal@yahoo.com; Caroline F Hilburn - hilburnc@onid.orst.edu; Stephanie Weitz - weitzs@onid.orst.edu; Wei-Siang Liau - wliau@csupomona.edu; Craig W LaMunyon - cwlamunyon@csupomona.edu; Dee R Denver* - denver@cgrb.oregonstate.edu

* Corresponding author

Published: 16 January 2009

BMC Evolutionary Biology 2009, 9:15 doi:10.1 186/147I-2/48-9-15
Received: 12 August 2008

Accepted: 16 January 2009

This article is available from: http://www.biomedcentral.com/I47I-2/48/9/15

(C) 2009 Lewis et al; licensee BioMed Central Ltd.

This is an Open Access article distributed under the terms of the Creative Commons Attribution License (http://creativecommons.org/licenses/by/2.0), which permits unrestricted use, distribution, and reproduction in any medium, provided the original work is properly cited.

\begin{abstract}
Background: As exemplified by the famously successful model organism Caenorhabditis elegans, nematodes offer outstanding animal systems for investigating diverse biological phenomena due to their small genome sizes, short generation times and ease of laboratory maintenance. Nematodes in the genus Panagrolaimus have served in comparative development and anhydrobiosis studies, and the Antarctic species $P$. davidi offers a powerful paradigm for understanding the biological mechanisms of extreme cold tolerance. Panagrolaimus nematodes are also unique in that examples of gonochoristic, hermaphroditic and parthenogenetic reproductive modes have been reported for members of this genus. The evolutionary origins of these varying reproductive modes and the Antarctic species $P$. davidi, however, remain enigmatic.

Results: We collected nuclear ribosomal RNA gene and mitochondrial protein-coding gene sequences from diverse Panagrolaimus species and strains, including newly discovered isolates from Oregon, to investigate phylogenetic relationships in this nematode genus. Nuclear phylogenies showed that the species and strains historically identified as members of Panagrolaimus constitute a paraphyletic group, suggesting that taxonomic revision is required for Panagrolaimus and related nematode lineages. Strain-specific reproductive modes were mapped onto the molecular phylogeny to show a single origin of parthenogenesis from a presumably gonochoristic ancestor. The hermaphroditic strains were all placed outside a major monophyletic clade that contained the majority of other Panagrolaimus nematodes. Phylogenetic analyses of mitochondrial sequences showed that substantial molecular and geographic diversity exists within the clade of parthenogenetic strains. The Antarctic species $P$. davidi was found to be very closely related to two Panagrolaimus strains from southern California. Phylogenetic and molecular clock analyses suggested that $P$. davidi and the California strain PSI579 shared a common ancestor in the very recent evolutionary past.
\end{abstract}

Conclusion: Our study provides a phylogenetic framework for understanding the evolutionary origins and diversification patterns of varying reproductive modes within Panagrolaimus and important insights into the origin of the Antarctic species $P$. davidi. Panagrolaimus offers a powerful nematode model for understanding diverse evolutionary phenomena including the evolution of asexuality and the adaptive evolution of extreme cold tolerance. 


\section{Background}

The nematode Caenorhabditis elegans has long served as an exceptional model system for understanding diverse biological phenomena such as development, neurobiology and genome structure [1-3]. Over the last decade nematodes in the Caenorhabditis genus have come to serve as increasingly powerful evolutionary models for studying diverse processes ranging from the mutation rate [4-6] to the evolution of reproductive mode transitions $[7,8]$ to speciation mechanisms [9-11]. Although C. elegans and C. briggsae were originally thought to share a common origin of hermaphroditic reproduction, more recent molecular phylogenetic approaches have shown independent origins of hermaphroditism for these two Caenorhabditis species [12]. There exists over one million genetically diverse nematode species outside of Caenorhabditis $[13,14]$ that are also coming to serve roles as models for other evolutionary phenomena. For example, nematodes in the genera Strongyloides and Rhabditophanes are growing models for understanding the evolution of parasitism [15]; Oscheius and Pristionchus nematodes offer strong models for investigating the evolution of developmental processes [16-19]. Multiple independent evolutionary transitions from gonochoristic to hermaphroditic reproduction have also been observed in the latter two nematode genera $[17,20]$ and a broad review of numerous rhabditid nematodes showed up to ten independent transitions to hermaphroditism [21].

Panagrolaimus nematodes have also played roles in comparative evolution of development studies $[16,22,23]$, and Panagrolaimus constitutes the only nematode genus in which three different reproductive modes (gonochoristic, hermaphroditic and parthenogenetic) have been reported. Well-studied parthenogenetic strains of Panagrolaimus include a number of isolates from North America (e.g. PS1579) as well as P. davidi from Antarctica; the latter has been shown to reproduce through meiotic parthenogenesis [24]. More recently this group has emerged as a model for understanding the physiological and molecular mechanisms underlying anhydrobiosis and extreme cold tolerance, due in part to the discovery of $P$. davidi in Antarctica. This species has evolved multiple strategies to deal with extreme cold including cryoprotective dehydration and the prevention and tolerance of intracellular freezing in adults and eggs [25-33]. A recent comparative analysis of extreme cold tolerance showed that the majority of individuals in experimental lab populations of $P$. davidi survived $-15^{\circ} \mathrm{C}$ treatments ( $\sim 80 \%$ survival) whereas much lower survival rates (all $<20 \%$ ) were observed in five other nematode species surveyed, including $P$. davidi's congener $P$. rigidus [25]. Panagrolaimus strain PS443 survived over eight years in a state of anhydrobiosis [34].
A previous phylogenetic analysis of Panagrolaimus species and strain relationships based on ribosomal RNA (rRNA) internal transcribed spacer regions showed that a number of strains in the same monophyletic clade as $P$. davidi were able to survive desiccation treatments at high rates whereas others outside that clade were much more sensitive to desiccation [35]. Although this initial study provided important insights into the evolutionary relationships of different Panagrolaimus strains and species, it was limited by inclusion of only eleven Panagrolaimus strains and the use of only one very distantly related outgroup (C. elegans). The evolutionary origins of varying reproductive modes across different Panagrolaimus lineages remain unknown, as do the origins of the lineage leading to the Antarctic species P. davidi.

Here we provide an extensive evolutionary analysis of thirty-one Panagrolaimus species and strains that utilizes both slowly-evolving nuclear loci and a faster-evolving mitochondrial locus to provide insights into the phylogenetic relationships of different lineages and to map the origins of parthenogenetic and hermaphroditic reproductive modes. Strain-specific reproductive modes were analyzed by assaying for the presence of sperm using microscopy techniques. To provide a rough estimate of the general timeline for $P$. davidi's arrival in Antarctica, we extended a molecular clock approach previously applied to Caenorhabditis [36] to approximate the divergence time between $P$. davidi and its closest known relative.

\section{Results and discussion Panagrolaimus strains and reproductive mode analysis} We initiated our study by requesting available Panagrolaimus strains from the Caenorhabditis Genetics Center and other helpful colleagues (see Table 1). Further, in the summer of 2007 we sampled soils from diverse locations around Oregon and discovered seven additional Panagrolaimus isolate strains from which laboratory cultures were generated and frozen stocks were made. A total of thirtyone strains were collected from our colleagues and the field that were successfully established in laboratory culture (Table 1). Four additional nematode strains isolated in Monroe, Oregon, USA (LD3, LD6, LD7 and LD8) were used for molecular analyses but were not kept in longterm laboratory culture. We are able to maintain the thirty-one Panagrolaimus strains listed in Table 1 at $25^{\circ} \mathrm{C}$ using standard OP50 Escherichia coli-seeded NGM plates. We are also able to cryogenically preserve all strains in glycerol solutions using standard $C$. elegans techniques [1], and to developmentally synchronize lab populations using the hypochlorite treatment method [37]. We discovered that multiple larval and adult developmental stages of Panagrolaimus nematodes survived cryogenic storage whereas in C. elegans only L1-stage larvae generally survive. We kept track of generation times and brood sizes for 
Table I: Panagrolaimus strains analyzed.

\begin{tabular}{|c|c|c|c|c|c|}
\hline Strain & Other names & Geographic origin & Source & Sperm & Reprod. \\
\hline AF36 & P. rigidus & Hungary? & CGC, $A B$ & ND & G \\
\hline AF40 & P. rigidus (Oscheius sp.) & Hungary? & CGC, $A B$ & ND & G \\
\hline BSS8 & P.detritophagus & Iceland & $A B$ & Yes & $\mathrm{H}$ \\
\hline BW287 & P.spl (C. briggsae) & Beijing, China & CGC, $A B$ & ND & $\mathrm{H}$ \\
\hline $\mathrm{CBI}$ & P. davidi & McMurdo Sound region, Antarctica & DW & No & $P$ \\
\hline DF5050 & P. superbus & Iceland & $A B$ & ND & G \\
\hline DL0050 & none & Portland, OR, USA & $\mathrm{DL}$ & No & $\mathrm{P}$ \\
\hline DL0072 & none & Corvallis, OR, USA & DL & No & $P$ \\
\hline DLOII7 & none & Portland, OR USA & $\mathrm{DL}$ & ND & G \\
\hline DLOI 28 & none & Salem, OR, USA & $\mathrm{DL}$ & ND & G \\
\hline DL0I37 & none & Corvallis, OR, USA & $\mathrm{DL}$ & No & $\mathrm{P}$ \\
\hline DL0I39 & none & Corvallis, OR, USA & DL & No & $P$ \\
\hline DLOI80 & none & Salem, OR, USA & DL & ND & G \\
\hline ESI & P. "brombeer" & Cologne, Germany & ES & ND & G \\
\hline ES2 & P. "brauwciler" & Cologne, Germany & ES & ND & $\mathrm{G}$ \\
\hline ES3 & P. "mais" & Eifel Mtn, Germany & ES & ND & $\mathrm{G}$ \\
\hline ES5 & P. "bornheim" & Bonn, Germany & ES & ND & G \\
\hline ES6 & P. sp. & Cologne, Germany & ES & ND & G \\
\hline JB05I & DF049 & Senegal & $\mathrm{JB}$ & No & $P$ \\
\hline $\mathrm{JBI} \mid 5$ & P. sp. & San Bernadino Mtns, CA, USA & $\mathrm{JB}$ & No & $P$ \\
\hline $\mathrm{JB}|3|$ & Eucephalobus sp.I & Baja California, Mexico & JB & No & $P$ \\
\hline JU765 & P. sp. & Guangxi, China & MAF & Yes & $\mathrm{H}$ \\
\hline PS443 & P. sp3 & Armenia & $A B, M A F$ & ND & G \\
\hline PSII59 & P. sp2 & N. Carolina, USA & $A B, M A F$ & No & $P$ \\
\hline PSII62 & P. sp. & Beijing, China & PS & No & $P$ \\
\hline PSI579 & P. sp4 & Huntington Gardens, CA, USA & CGC, $A B$ & ND & $P$ \\
\hline PSI732 & P. sp5 & Sierra Mtns, CA, USA & PS & Yes & $\mathrm{H}$ \\
\hline PSI806 & none & Huntington, CA, USA & PS & No & $\mathrm{P}$ \\
\hline PS3966 & none & Pasadena, CA, USA & PS & No & $P$ \\
\hline SNIO3 & none & Susanville, CA, USA & $A B$ & No & $P$ \\
\hline none & P.paetzoldi & Westerscheldt, Netherlands & $A B$ & ND & G \\
\hline
\end{tabular}

Strains sources are: JB, James G. Baldwin; AB, Ann Burnell; DL, Denver lab; MAF, Marie-Anne Félix; ES, Einhard Schierenberg; PS, Paul W. Sternberg; DW, David Wharton. Modes of reproduction (Reprod.) are: $G$, gonochoristic; $H$, hermaphroditic; $P$ parthenogenetic.

two Panagrolaimus strains (PS1579 and PS1159 - both parthenogenetic) and found that they experience generation times of approximately seven days and brood sizes of 300-400 eggs under the aforementioned laboratory culturing conditions. Thus, Panagrolaimus offers many of the same basic attractive features that have contributed to the outstanding success of the C. elegans system. Furthermore, RNA interference-mediated gene silencing has recently been documented to be effective in P. superbus and the parthenogenetic Panagrolaimus strain PS1162 [38].

We initially distinguished between gonochoristic and selffertile Panagrolaimus strains by carrying out self-fertility tests whereby early larval stage nematodes were individually transferred to plates and then allowed to develop in isolation. Strains repeatedly unable to carry out self-fertilization were defined as gonochoristic - in all cases, mass populations of strains found to be gonochoristic by this criterion were also observed to contain large numbers of mating males. Our definitions of gonochoristic strains were also consistent with previous independent observations $[16,23,35]$.

For all self-fertile strains, we tested for the presence or absence of sperm using DIC/epifluorescence microscopy techniques (see Methods for details). Three self-fertile Panagrolaimus species/strains (JU765, PS1732, P. detritophagus) were observed to produce sperm (Figure 1) - we consider these to most likely be hermaphroditic, though we cannot rule out the possibility that the sperm is only used for egg activation and does not contribute genetic material. For all other self-fertile Panagrolaimus strains examined, no sperm was detected and we consider them to be parthenogenetic (Figure 2). In mass laboratory populations, males are frequently observed for $P$. detritophagus and occasionally observed for JU765 and PS1732. We have not observed males in any of the parthenogenetic strains, though a focused study on male frequencies has not yet been carried out. Males were observed to be abundant in field populations of the Antarctic species $P$. davidi 

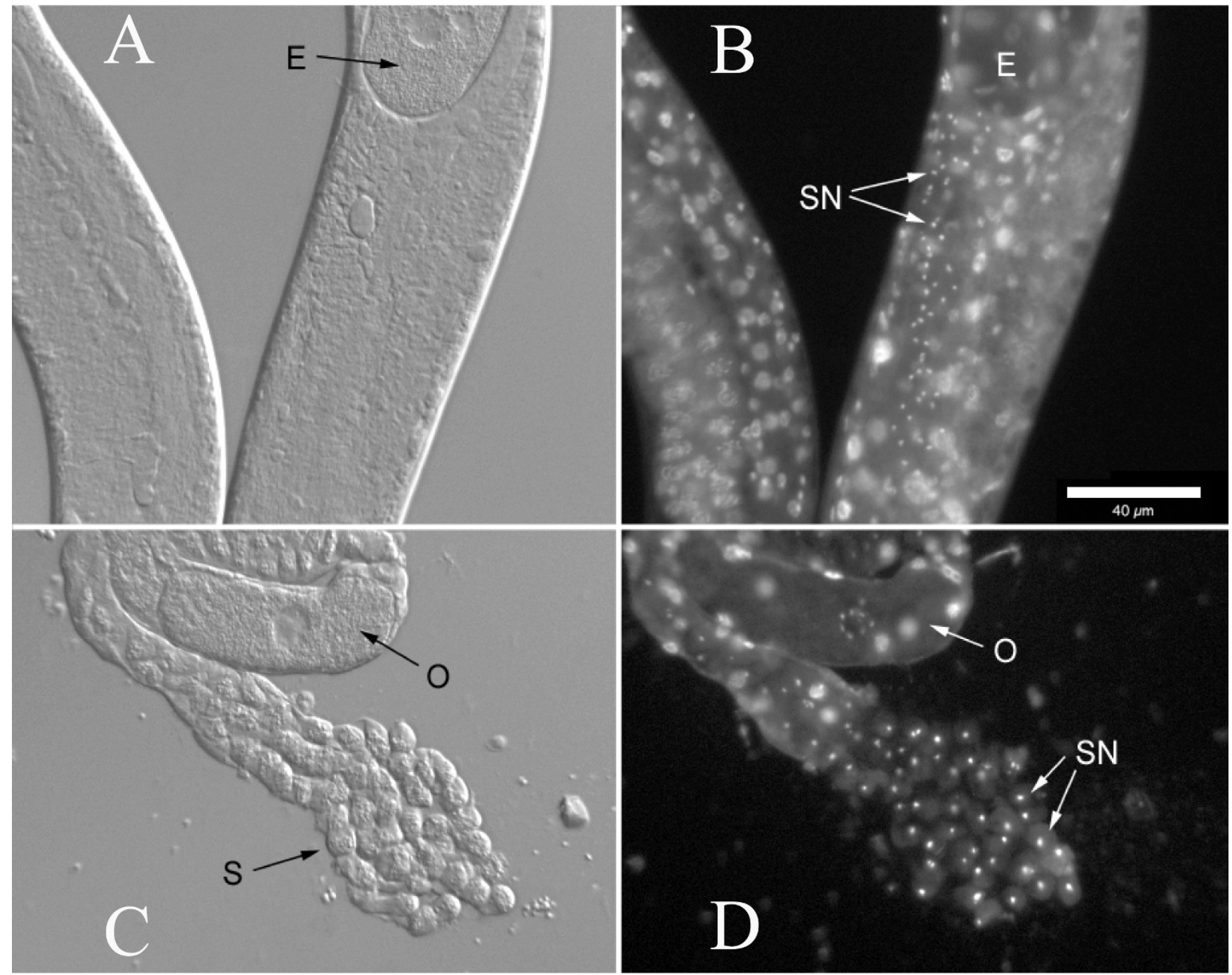

\section{Figure I}

Identification of sperm and sperm nuclei from hermaphroditic Panagrolaimus strain JU765. DIC imaging was used to identify sperm (left, panels $A$ and $C$ ) and epifluorescence imaging was used to identify characteristically compact sperm nuclei (right, panels B and D). See Methods for details. A and B show nematode midbodies; C and D show a dissected spermatheca. Ann egg is indicated by "E", sperm nuclei by "SN", oocytes by "O", and sperm by "S". The scalebar represents $40 \mu \mathrm{m}$.

whereas parthenogenetic females were observed to dominate long-term lab cultures [39].

\section{Panagrolaimus evolutionary relationships}

We applied a molecular phylogenetic approach to understand the evolutionary relationships of Panagrolaimus species and strains, and to map the origins of reproductive mode transitions in this nematode genus. We PCR-amplified and directly sequenced segments of the $18 \mathrm{~S}$ and $28 \mathrm{~S}$ rRNA genes from genomic DNA samples derived from each of the thirty-one Panagrolaimus strains (see Methods). Amplified sequences were subjected to multiple alignment using ClustalW in the program MEGA4 [40]; subsequently numerous orthologous outgroup sequences representing diverse nematode taxa of varying evolutionary distances from Panagrolaimus were also included in the alignment (sequences obtained from Genbank). After making minor corrections to the alignment based on visual inspection, we performed phylogenetic analyses in MEGA4 using both neighbor-joining (NJ) and maximum parsimony (MP) approaches (see Methods). A total of 436 aligned nucleotide sites were analyzed for 18S rRNA; 623 aligned nucleotide sites were analyzed for $28 \mathrm{~S}$ rRNA. Concatenated $18 \mathrm{~S}$ and $28 \mathrm{~S}$ sequences $(1,059$ sites total) were used for phylogenetic analyses. Additional analyses involved a segment (778 aligned nucleotide sites) of the 

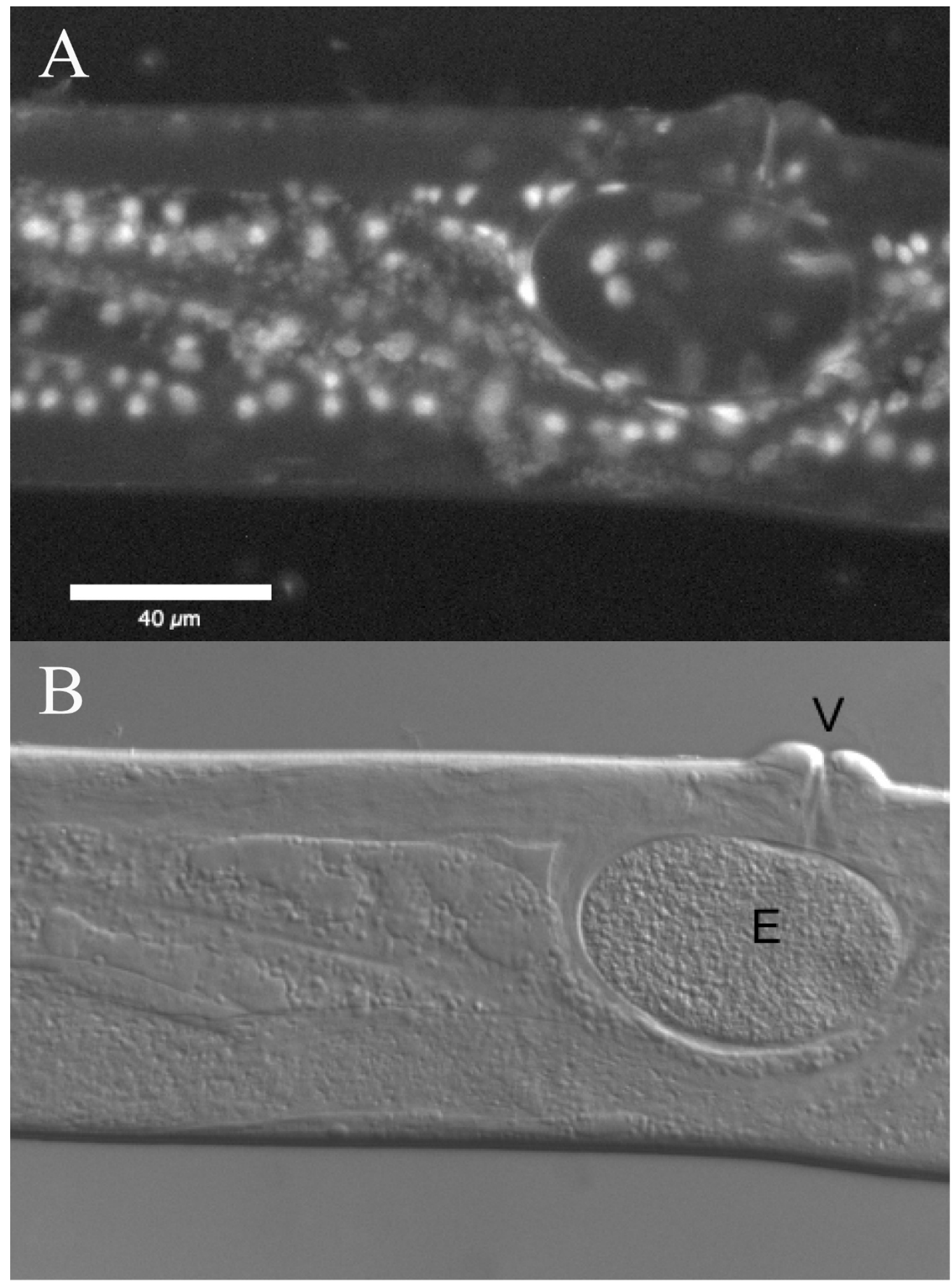

\section{Figure 2}

Lack of sperm and sperm nuclei from parthenogenetic Panagrolaimus strain JB I I 5. DIC imaging was used to search for sperm and epifluorescence to search for compact sperm nuclei (none were observed). See Methods for details. An egg is indicated by "E" and the vulva is indicated by " $\mathrm{V}$ ". The scalebar represents $40 \mu \mathrm{m}$. 
more rapidly evolving mitochondrial NADH dehydrogenase subunit 5 (ND5) gene to independently test the results of nuclear rRNA analyses and further analyze molecular diversification patterns. ND5 sequences were successfully obtained for all Panagrolaimus except DL0139 (Corvallis, Oregon, USA) and ES6 (Cologne, Germany). As with the nuclear loci, aligned ND5 sequences were subject to MP and NJ phylogenetic analyses in MEGA4 (see Methods). One thousand bootstrap replicates were performed in each analysis to estimate confidence in nodes resulting from the analyses.

$\mathrm{NJ}$ and MP analyses of the aligned nuclear rRNA data yielded highly congruent bootstrap consensus trees (Figure 3, Additional files 1,2). Although there was a single well-supported monophyletic clade (referred to herein as Clade PI) that exclusively contained 25/31 Panagrolaimus sequences analyzed, four other Panagrolaimus sequences (JU765, PS1732, P. detritophagus BSS8, P. paetzoldi) were included with a separate monophyletic clade, referred to herein as Clade PII, also containing sequences from Halicephalobus gingivalis [41] and related isolates. The sequence from strain AF40 was grouped with the rhabditid Oscheius tipulae and the sequence from strain BW287 was grouped with C. elegans and its congener C. briggsae. A nearly complete mitochondrial genome sequence was previously generated for BW287 which confirmed that it is a strain of C. briggsae [4]. These two strains historically identified as members of this genus (AF40, BW287) are clearly members of the very distantly related rhabditid group of nematodes and likely constitute simple accidental cases of misidentification or laboratory contamination.

Our results suggest that a taxonomic revision is required for Panagrolaimus since those strains historically identified as members of the genus do not compose a monophyletic group. There are two approaches to solving this dilemma given the phylogeny shown in Figure 3: one would be to remove the four PII Panagrolaimus strains from the genus rendering PI the monophyletic Panagrolaimus genus; the second would be to reassign Halicephalobus gingivalis and related nematodes to the genus Panagrolaimus rendering the monophyletic clade containing PI and PII the Panagrolaimus genus. Proper systematic revision of this nematode group and its relatives, though clearly necessary, will require a more broad-based analysis involving additional nematode genera (e.g. Panagrellus, Panagrobelus, Plectonchus, Turbatrix), more genetic markers and reconsideration of morphological features [42]. Rather than proposing specific taxonomic revisions for the present study whose focus is outside of these specific systematic issues, we will maintain the historical Panagraimus identifiers listed in Table 1 and refer to clades PI and PII (Figure 3) to distinguish between different Panagrolaimus subgroups.

\section{Evolution of reproductive mode transitions}

We next mapped reproductive modes onto the rRNA consensus phylogeny to identify the evolutionary origins of reproductive mode transitions. Within Clade PI we identified a monophyletic subclade (referred to herein as Clade PIp) that exclusively contained all of the parthenogenetic Pangrolaimus strains and species (Figure 3 ). This observation indicates a single evolutionary origin of parthenogenetic reproduction in Panagrolaimus.

The remaining Clade PI species and strains outside of Subclade PIp were all gonochoristic - Additional file 3 provides a summary of knowledge on species boundaries among the PI gonochoristic strains.

The three hermaphroditic Panagrolaimus species and strains ( $P$. detritophagus, JU765, PS1732) were all placed outside of Clade PI and were included in a separate monophyletic clade (PII) containing one gonochoristic Panagrolaimus species $(P$. paetzoldi) along with the opportunistic equine (and sometimes human) pathogen Halicephalobus gingivalis [41] and related nematodes (LD3, LD6, LD7, LD8) that we did not maintain in long-term culture. The nuclear phylogeny suggests a single origin of hermaphroditism in PII, although the small number of PII Panagrolaimus strains available for analysis precludes any strong conclusions.

In the PI clade there appears to have been a single evolutionary transition to parthenogenesis; among the PII Panagrolaimus strains the phylogeny suggests a single transition to hermaphroditism. By contrast, there is evidence for at least five independent transitions to hermaphroditism in Pristionchus [20], three in Oscheius [17], and three in Caenorhabditis (K. Kiontke, pers. comm.). Although this might superficially suggest a reduced plasticity in sex determination mechanisms regulating hermaphroditic versus female development in Panagrolaimus versus other well-studied nematode genera, Panagrolaimus likely suffers more than the other genera in terms of taxon undersampling - our observations in PII are based on only four Panagrolaimus strains. Although a more rigorous sampling of nematodes in the PII clade is required for a robust understanding of reproductive mode transitions in this group, it is interesting to note that $H$. gingivalis in PII reproduces parthenogenetically in infected horses [41]. PII thus might represent a nematode group of exceptional sex determination plasticity able to explore multiple reproductive mode strategies.

\section{Molecular diversification in the parthenogens}

In addition to illuminating the broad-based evolutionary relationships of all nematodes historically placed in the Panagrolaimus genus, our rRNA analyses also provided evidence for subdivision of the PIp clade of parthenogens 


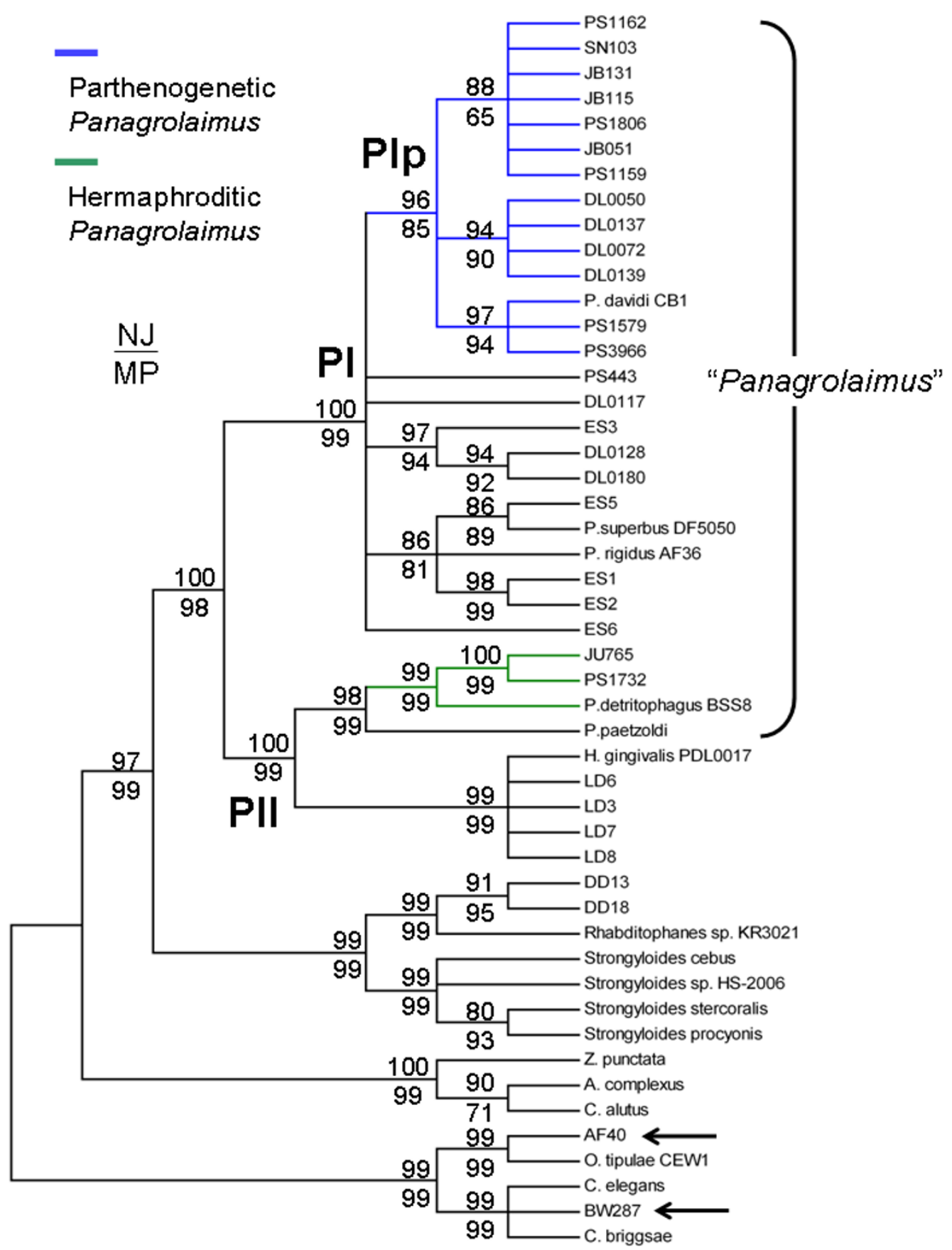

Figure 3

Nuclear rRNA gene phylogeny for Panagrolaimus. Cladogram shown is a $80 \%$ bootstrap consensus tree for $\mathrm{NJ}$ analysis of aligned I8S and 28S rRNA sequences from Panagrolaimus and select outgroup nematode species and strains. The MP phylogeny was highly congruent with the NJ phylogeny shown - see Additional files I and 2. Node-specific bootstrap values (I,000 replicates for each analysis) are shown, with NJ values over MP values. All strains and species historically considered as members of the Panagrolaimus genus are indicated by the brackets. Arrows denote the positions of two rhabditid strains initially misidentified as Panagrolaimus. Blue lines indicate parthenogenetic strains and green lines indicate hermaphroditic strains of Panagrolaimus. $H$. gingivalis also reproduces parthenogenetically in infected horses. DDI3 and DDI8 are two strains isolated from Newport, OR USA that are closely related to Rhabditophanes sp. KR302I. All other outgroup sequences were retrieved from Genbank. 
into three subclades (Figure 3). To further investigate divergence in the PIp clade, we analyzed a segment of the more rapidly evolving ND5 gene from a subset of Panagrolaimus strains to independently test the results of nuclear rRNA analyses and further analyze diversification within the PIp clade of parthenogens. Sequences were successfully obtained for 13/14 of the PIp parthenogens - we were repeatedly unable to PCR amplify the ND5 fragment from DL0139 (Corvallis, Oregon, USA).

The mitochondrial ND5 gene NJ phylogeny (Figure 4, Additional file 4) was highly congruent with the nuclear rRNA phylogeny (Figure 3). The PI, HI and PIp clades were each supported with strong ( $>80 \%$ ) boostrap support in NJ analysis of mtDNA sequence. Clade PIp was also monophyletic in MP analysis of mtDNA, though with low boostrap support; similarly Clade PII was monophyletic in MP mtDNA analysis with low bootstrap support (see Figure 4). Clade PI, however, was strongly supported in both $\mathrm{NJ}$ and MP analyses of the ND5 sequences; furthermore, topological arrangement of all strain analyzed was identical for NJ and MP analyses of ND5 (Additional files 4, 5). The distribution of parthenogenetic strains in Clade PIp subclades in ND5 phylogenetic analyses was the same for that of nuclear rRNA analyses. Among the thirteen PIp Panagrolaimus parthenogenetic strains considered in mtDNA analysis, seven unique ND5 haplotypes were observed. One haplotype was shared by six strains - three were from California, USA, the fourth was from North Carolina, USA, the fifth was from Baja California, Mexico and the sixth was from Senegal. The sharing of a mtDNA haplotype between strains from North America with one from the west coast of Africa might indicate that Panagrolaimus strains are able to very rapidly disperse across large geographic distances, as has been proposed for C. elegans where identical mitochondrial genome haplotypes have also been observed for geographically disparate isolates [43]. Like Caenorhabditis and Pristionchus $[47,48]$, Panagrolaimus has recently been described to associate with arthropod hosts that might aid in its geographic dispersal [49]. The three PIp Oregon strains analyzed formed a well-supported monophyletic group and all had highly similar ND5 haplotypes. Strain PS1162 from China had a unique ND5 haplotype. PS1579 and PS3966 were found to share a haplotype. P. davidi CB1 and these two California strains (PS1579, PS3966) were again found to form a well-supported monophyletic clade and share highly similar ND5 sequences (Figure 4), suggesting that the Antarctic strain $P$. davidi CB1 and these strains from California shared a common ancestor in the not-too-distant past.

\section{Arrival and evolution of $\mathbf{P}$. davidi in Antarctica}

Two independent molecular phylogenies presented here (Figure 3, Figure 4) show very close evolutionary relation- ships between two PIp parthenogens from southern California (PS1579, PS3966) and P. davidi CB1 from Antarctica. To extend upon the phylogenetic results, we applied a molecular clock approach to estimate the number of nematode generations that separate PS1579 and $P$. davidi $\mathrm{CB} 1$. Under the neutral theory of molecular evolution [44], the rate of mutation is expected to be equal to the rate of substitution at neutral sites, and the time to the most recent common ancestor (TMRCA) for any two taxa for which there is relevant DNA sequence data can be estimated by the equation $\mathrm{T}=K /(2 \mu)$ where $\mathrm{T}$ is the time to the most recent common ancestor, $K$ is a measure of the neutral molecular divergence between the compared taxa and $\mu$ is the mutation rate [45]. For the nematode C. elegans, there are direct estimates of the pergeneration nuclear and mtDNA mutation rates based on mutation-accumulation line systems $[5,6]$. The nuclear rate estimate was recently employed in an analysis of divergence times among Caenorhabditis species utilizing an internally calibrated molecular clock approach [36]. We applied a similar (though externally calibrated) approach here, assuming that the Panagrolaimus mtDNA $\mu$ is the same as that estimated for $C$. elegans, to estimate the TMRCA for PS1579 from California and P. davidi from Antarctica.

To provide a broader mtDNA data set for evolutionary rate analysis, we collected additional mtDNA protein-coding gene sequences for PS1579 and P. davidi CB1; data examined includes sequences from Cytochrome b, Cytochrome Oxidase I (COI), ND1 and ND5; 2,247 bp, 749 codons total. We employed DnaSP v4.1 [46] to calculate the rate of silent-site substitution $\left(d_{S}\right)$, a value often used to estimate neutral evolutionary rates, in the concatenated protein-coding gene sequence pairwise alignment - a $d_{S}$ value of 0.027 was estimated from the PS1579-P. davidi alignment. Employing the mtDNA $d_{S}(0.027)$ estimate suggests a rough TMRCA value of 140,206 nematode generations for PS1579 and $P$. davidi. Translating the number of nematode generations into actual (chronological) time is problematic due to uncertainties in the numbers of generations experienced by Panagrolaimus in the wild - a general problem for nematodes [36]. Although PS1579 was observed to experience generation times of seven days in the lab at $25^{\circ} \mathrm{C}$ under food-rich conditions, suggesting approximately fifty-two generations per year, massive environmental differences between the lab and nature (e.g. temperature, food supply type and abundance) almost certainly affect the number of Panagrolaimus generations per year in nature. In particular, $P$. davidi's native Antarctic environment likely provides conditions where nematodes are able to reproduce for a few months (if not weeks) per year [39]. By contrast, the much warmer climate of southern California experienced by PS1579 likely enables a much greater number of generations per year. 


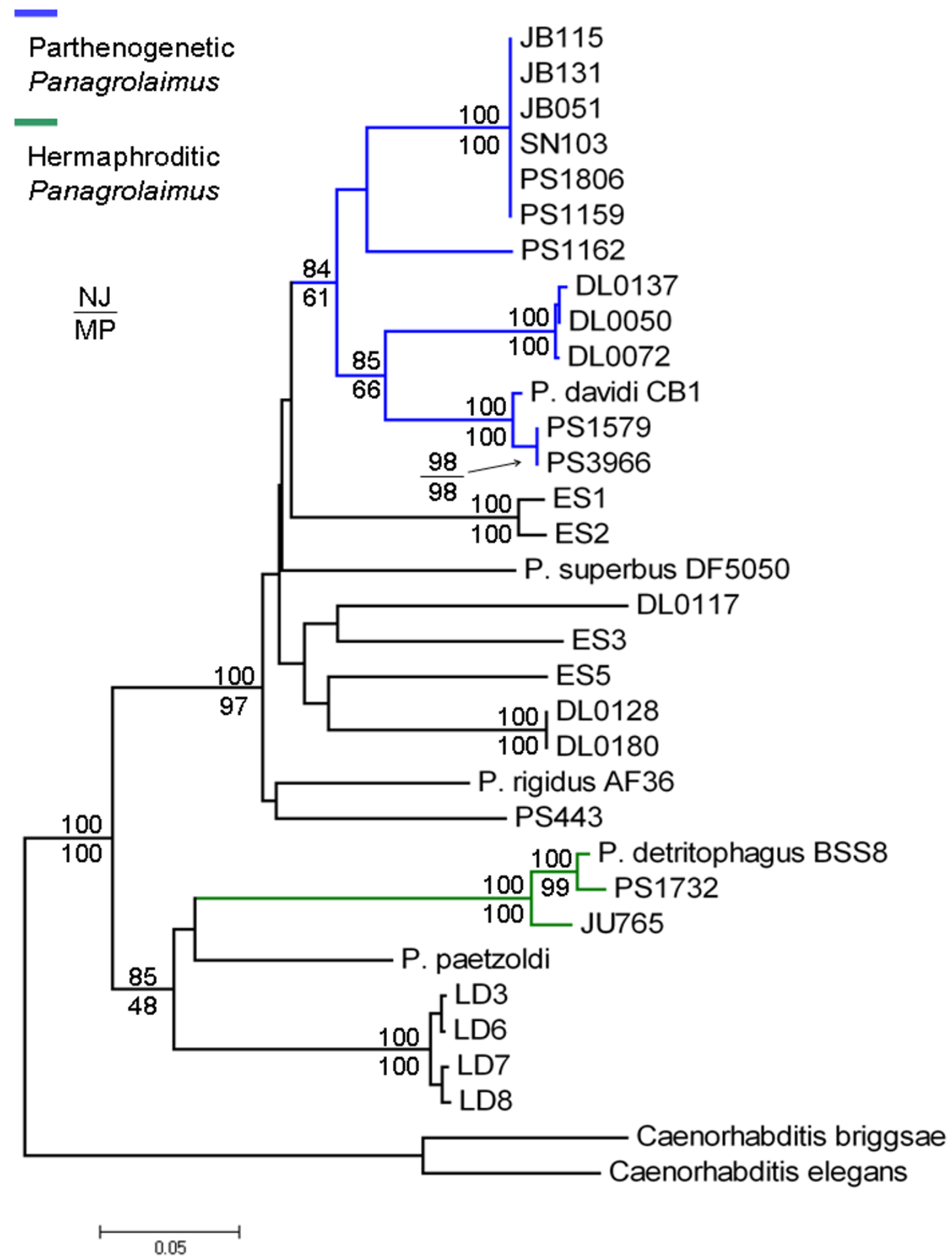

\section{Figure 4}

Mitochondrial ND5 gene phylogeny for Panagrolaimus. Phylogram shows relationships among a subset of Panagrolaimus ND5 sequences (those able to be successfully amplified) inferred from NJ phylogenetic analysis (see Methods). Scale bar shows 0.05 substitutions per site. Bootstrap values resulting from NJ and MP analyses are shown for select nodes (e.g. those supporting PI, Plp and HI). Additional files 4 and 5 show complete phylograms with all bootstrap values for NJ and MP analyses, respectively. Blue lines indicate parthenogenetic strains and green lines indicate hermaphroditic strains. 
Additional file 6 shows the relationship between TMRCA estimates (in years) for PS1579 and P. davidi and assumed numbers of nematode generations per year. If PS1579 and $P$. davidi have both experienced as few as ten generations per year since their divergence, our analyses based on $d_{S}$ roughly approximate a TMRCA of only $\sim 14,000$ years. Even if these two taxa have each experienced only one generation per year since their divergence, a TMRCA of only $\sim 140,000$ years is approximated. These TMRCA measures should be interpreted with great caution, however, given the untested assumptions underlying these particular molecular clock analyses - for example, that mtDNA mutation rates are the same for $C$. elegans and Panagrolaimus and that the rate of mutation equals the rate of silent-site substitution. Nonetheless, the very close genetic distances between PS1579, PS3966 and P. davidi CB1 along with the phylogenies presented in Figures 3 and 4 also generally suggest that $P$. davidi colonized Antarctica in the very recent past.

Did $P$. davidi adaptively evolve extreme cold tolerance after its arrival in Antarctica? Although a current comparative cryotolerance study involving $P$. davidi showed that this species was unique in its extreme cold tolerance as compared to other nematode species examined, the other nematodes used for comparison in the study were very distantly related to $P$. davidi - the closest relative was $P$. rigidus that our phylogenetic and genetic distance analyses show is very distantly related to $P$. davidi. Future comparative cryotolerance studies involving $P$. davidi's closelyrelated parthenogen relatives (e.g. PS1579) would shed light on whether extreme cold tolerance is a feature unique to $P$. davidi, or if it is shared by its closely-related parthenogenetic relatives. If the latter scenario is true, it would suggest that $P$. davidi arrived in Antarctica in a "preadapted", cryotolerant state. If the latter scenario is not true, it would suggest that $P$. davidi was able to very rapidly adapt to the harsh Antarctic environment.

\section{Conclusion}

Our study sheds light on the evolutionary relationships of Panagrolaimus nematodes, showing that the traditional genus is paraphyletic. Our analyses suggest a single origin of parthenogenesis in Panagrolaimus and provide evidence that $P$. davidi arrived in Antarctica in the very recent evolutionary past. This nematode genus offers an outstanding model for analyzing the evolutionary and biological causes of transitions from gonochoristic to parthenogenetic reproduction, and the adaptive evolution of extreme cold tolerance. We also note here that a brief soil survey effort in northwest Oregon performed by a single undergraduate student (C. F. H.) resulted in the discovery two probable new gonochoristic species of Panagrolaimus (DL0117 and DL0128/DL0180 - see Additional file 3) and a new major clade of PIp parthenogens. The world- wide diversity of Panagrolaimus and other nematode species in the soil and other ecologies likely remains vastly undersurveyed, being a key limiting factor in our understanding of evolutionary processes in this diverse animal phylum.

\section{Methods \\ Strain sources and maintenance}

Thirty-one strains of Panagrolaimus were obtained from various sources for analysis (Table 1 ). All nematodes were cultured on standard NGM agarose plates, seeded with the OP50 strain of E. coli as a food source. Nematodes were maintained at $25^{\circ} \mathrm{C}$ in Percival incubators, and transferred to fresh plates once a week. Frozen stocks were created for each Panagrolaimus strain following standard C. elegans freezing protocols [1]. C. elegans standard bleaching protocols for developmental synchronization [37] were also applied to the Panagrolaimus strains.

\section{Microscopy methods for sperm identification}

At least 10 gravid worms from each strain were placed into a droplet of ethanol on a microscope slide, and the ethanol was allowed to evaporate completely. A $10 \mu \mathrm{l}$ drop of PBS containing $20 \mu \mathrm{g} / \mathrm{ml}$ of DAPI (4',6-diamidino-2-phenylindole) was added to the ethanol-fixed worms to label the nuclei [50]. A coverslip was placed over the labeled worms, and they were observed under epifluorescence. Sperm were identified by their characteristic compact nuclei. When sperm nuclei were detected for a particular worm species, the identification was verified by dissecting additional worms from that species in Sperm Medium (SM1: $50 \mathrm{mM}$ HEPES pH7.0, $50 \mathrm{mM} \mathrm{NaCl}, 25 \mathrm{mM} \mathrm{KCl}, 1$ $\mathrm{mM} \mathrm{MgSO}_{4}, 5 \mathrm{mM} \mathrm{CaCl}_{2}, 20 \mathrm{mM}$ Glucose pH7.0) containing DAPI $(20 \mu \mathrm{g} / \mathrm{ml})$. The sperm were identified under differential interference contrast (DIC) optics, and their nuclei were observed under epifluorescence.

\section{PCR amplification and DNA sequencing}

Nematode populations were allowed to expand until food sources were exhausted, after which the worms were harvested for DNA extraction, using previously-established protocols for C. elegans [43]. For nuclear loci, standard polymerase chain reaction (PCR) amplifications for $18 \mathrm{~S}$ and $28 \mathrm{~S}$ rRNA gene sequences were performed in $50 \mu \mathrm{L}$ reactions as previously described [43]. The $18 \mathrm{~S}$ rRNA fragment was amplified using primers $18 \mathrm{~A}$ and $26 \mathrm{~B}$ [51] and the 28S rRNA fragment was amplified using primers \#537F and \#531R [42]. The mitochondrial segment analyzed across multiple Panagrolaimus strains (ND5 gene) was amplified using the Roche Expand Long Range PCR kit with primers $39 \mathrm{~F}$ and $58 \mathrm{R}$ that are nearly universal nematode mtDNA PCR primers [52]. Additional mtDNA segments exclusively analyzed in PS1579 and P. davidi CB1 resulted from two additional PCR amplifications: COIF to 40R and PS5F to 36R. Universal nematode 
mtDNA primers were again employed, with the exception of PS5F that we designed specific to PS1579 mtDNA sequences. All primer sequences are available upon request. PCR products were purified using solid phase reversible immobilization techniques [53] and then used as template for cycle sequencing. PCR primers were used for sequencing, along with internal primers when necessary. Cycle sequencing was carried out by 25 cycles of denaturation at $96^{\circ} \mathrm{C}$ for 30 seconds, annealing at $50^{\circ} \mathrm{C}$ for 15 seconds, and extension at $60^{\circ} \mathrm{C}$ for 4 minutes. Unincorporated dye terminators were removed by ethanol precipitation and DNA sequencing reactions were analyzed using an $\mathrm{ABI} 3730$ capillary sequencer at the OSU Center for Genome Research and Biocomputing. New DNA sequences used in this study were deposited in Genbank under accession numbers FI590596-FI591048.

\section{Alignment and evolutionary analyses}

Alignment and phylogenetic analysis procedures were carried out identically for nuclear and mtDNA sequences. DNA sequence alignments and phylogenetic analyses were performed using MEGA4 [40]. Alignments were done using the ClustalW feature offered by MEGA4 - gap opening penalties were set at 15 and gap extension penalties at 6.66. The IUB DNA weight matrix was utilized. After alignment, the ends of sequences were trimmed off to create aligned sequences of equal character length and then the $18 \mathrm{~S}$ and $28 \mathrm{~S}$ rDNA sequences were joined for subsequent phylogenetic analyses. The ND5 mtDNA sequences were analyzed independently from the nuclear sequences. After automatic alignment, minor manual adjustments to the alignment were made prior to phylogenetic analysis. MEGA4 was also applied for MP and NJ phylogenetic analyses. The maximum-composite likelihood molecular evolution model was used for neighborjoining analyses. 1,000 bootstrap replicates were performed for all phylogenetic testing. We estimated $d_{S}$ values from PS1579-P. davidi CB1 mtDNA alignment files using DNAsp v4.1 [46].

\section{Abbreviations}

$d_{S}$ : rate of silent-site substitution; mtDNA: mitochondrial DNA; MP: maximum parsimony; NJ: neighbor joining; rRNA: ribosomal RNA; TMRCA: time to the most recent common ancestor

\section{Authors' contributions}

SCL performed all mitochondrial sequencing experiments and evolutionary analyses, and contributed to manuscript writing; LAD contributed to nuclear sequencing experiments and evolutionary analyses, reproductive mode analyses and manuscript writing; $\mathrm{CH}$ contributed to nuclear sequencing experiments, set up the mating tests and discovered the Oregon Panagrolaimus strains; SW contributed to strain maintenance and reproductive mode analyses; W-SL contributed to microscopy experiments; CWL contributed to microscopy experiments; DRD conceived the study and contributed to evolutionary analyses and manuscript writing. All authors read and approved the manuscript.

\section{Additional material}

\section{Additional file 1}

NJ phylogram for nuclear rRNA sequences. Complete bootstrap consensus phylogram for NJ analysis, including bootrap support across all nodes, is shown. Scale bar shows 0.01 substitutions per site.

Click here for file

[http://www.biomedcentral.com/content/supplementary/14712148-9-15-S1.tiff]

\section{Additional file 2}

MP phylogram for nuclear rRNA sequences. Complete bootstrap consensus phylogram for MP analysis, including bootrap support across all nodes, is shown. Scale bar shows 50 substitutions.

Click here for file

[http://www.biomedcentral.com/content/supplementary/14712148-9-15-S2.tiff]

\section{Additional file 3}

Species boundaries among the PI gonochoristic strains. The note provides a brief description of knowledge on species boundaries among gonochoristic Panagrolaimus the PI clade, and a table of nuclear and mitochondrial genetic distances between these strains.

Click here for file

[http://www.biomedcentral.com/content/supplementary/14712148-9-15-S3.doc]

\section{Additional file 4}

NJ phylogram for mitochondrial ND5 sequences. Complete bootstrap consensus phylogram for NJ analysis, including bootrap support across all nodes, is shown. Scale bar shows 0.05 substitutions per site.

Click here for file

[http://www.biomedcentral.com/content/supplementary/14712148-9-15-S4.tiff]

\section{Additional file 5}

MP phylogram for mitochondrial ND5 sequences. Complete bootstrap consensus phylogram for MP analysis, including bootrap support across all nodes, is shown. Scale bar shows 20 substitutions.

Click here for file

[http://www.biomedcentral.com/content/supplementary/14712148-9-15-S5.tiff]

\section{Additional file 6}

TMRCA estimates for PS1579 and P. davidi CB1. TMRCA estimate (in years) is shown on the $y$ axis assumed number of nematode generations per year is shown on the $x$ axis. Relationships are shown on a log-log plot. See Methods and main text for details.

Click here for file

[http://www.biomedcentral.com/content/supplementary/14712148-9-15-S6.tiff] 


\section{Acknowledgements}

We thank James G. Baldwin, Ann M. Burnell, Marie-Anne Félix, Einhard Schierenberg, Paul W. Sternberg, David Wharton and the Caenorhabditis Genetics Center for providing nematode strains. Thanks to Bobby Babra and Dana K. Howe for laboratory assistance and helpful conversations, and to Mark Dasenko at the OSU Center for Genome Research and Biocomputing for DNA sequencing assistance. We also thank two anonymous reviewers for their helpful comments. Special thanks to James G. Baldwin and Steven A. Nadler for comments and advice on nematode systematics. This work was supported by the OSU Computational and Genome Biology Initiative startup support to DRD and from the National Institutes of Health (Award 5 S06 GM053933-10) to CWL. CFH is supported by an OSU Undergraduate Research, Innovation, Scholarship \& Creativity grant.

\section{References}

I. Wood WB: The Nematode Caenorhabditis elegans. Cold Spring Harbor, NY: Cold Spring Harbor Laboratory Press; 1988.

2. Genome sequence of the nematode $C$. elegans: a platform for investigating biology. Science 1998, 282(5396):2012-2018.

3. Hillier LW, Coulson A, Murray JI, Bao Z, Sulston JE, Waterston RH: Genomics in C. elegans: so many genes, such a little worm. Genome Res 2005, I5(12): 1651-1660.

4. Howe DK, Denver DR: Muller's Ratchet and compensatory mutation in Caenorhabditis briggsae mitochondrial genome evolution. BMC Evol Biol 2008, 8:62.

5. Denver DR, Morris K, Lynch M, Thomas WK: High mutation rate and predominance of insertions in the Caenorhabditis elegans nuclear genome. Nature 2004, 430(7000):679-682.

6. Denver DR, Morris K, Lynch M, Vassilieva LL, Thomas WK: High direct estimate of the mutation rate in the mitochondria genome of Caenorhabditis elegans. Science 2000, 289(5488):2342-2344.

7. Braendle C, Felix MA: Sex determination: ways to evolve a hermaphrodite. Curr Biol 2006, I6( I 2):R468-47I

8. Hill RC, de Carvalho CE, Salogiannis J, Schlager B, Pilgrim D, Haag ES: Genetic flexibility in the convergent evolution of hermaphroditism in Caenorhabditis nematodes. Dev Cell 2006, I0(4):531-538.

9. Baird SE: Haldane's rule by sexual transformation in Caenorhabditis. Genetics 2002, 161(3):1349-1353.

10. Baird SE, Yen WC: Reproductive isolation in Caenorhabditis: terminal phenotypes of hybrid embryos. Evol Dev 2000, 2(1):9-15.

II. Seidel HS, Rockman MV, Kruglyak L: Widespread genetic incompatibility in $\mathrm{C}$. elegans maintained by balancing selection. Science 2008, 319(5863):589-594.

12. Kiontke K, Gavin NP, Raynes Y, Roehrig C, Piano F, Fitch DH: Caenorhabditis phylogeny predicts convergence of hermaphroditism and extensive intron loss. Proc Natl Acad Sci USA 2004, I 0 I(24): $9003-9008$.

13. Lambshead PJ, Brown CJ, Ferrero TJ, Hawkins LE, Smith CR, Mitchell $\mathrm{NJ}$ : Biodiversity of nematode assemblages from the region of the Clarion-Clipperton Fracture Zone, an area of commercial mining interest. $B M C$ Ecol 2003, 3:1.

14. Meldal BH, Debenham NJ, De Ley P, De Ley IT, Vanfleteren IR, Vierstraete AR, Bert W, Borgonie G, Moens T, Tyler PA, et al.: An improved molecular phylogeny of the Nematoda with special emphasis on marine taxa. Mol Phylogenet Evol 2007, 42(3):622-636.

15. Dorris M, Viney ME, Blaxter ML: Molecular phylogenetic analysis of the genus Strongyloides and related nematodes. Int J Parasitol 2002, 32(12): 1507-1517.

16. Felix MA, De Ley P, Sommer RJ, Frisse L, Nadler SA, Thomas WK, Vanfleteren J, Sternberg PW: Evolution of vulva development in the Cephalobina (Nematoda). Dev Biol 2000, 22 I (I):68-86.

17. Felix MA: Oscheius tipulae. WormBook 2006:I-8.

18. Sommer RJ: Evolution of development in nematodes related to C. elegans. WormBook 2005:I-17.

19. Delattre M, Felix MA: Microevolutionary studies in nematodes: a beginning. Bioessays 200I, 23(9):807-8I9.

20. Mayer WE, Herrmann M, Sommer RJ: Phylogeny of the nematode genus Pristionchus and implications for biodiversity, bioge- ography and the evolution of hermaphroditism. BMC Evol Biol 2007, 7:104.

21. Kiontke $\mathrm{K}$, Fitch $\mathrm{DH}$ : The phylogenetic relationships of Caenorhabditis and other rhabditids. WormBook 2005: I-II.

22. Felix MA, Sternberg PW: Two nested gonadal inductions of the vulva in nematodes. Development I997, I 24(I):253-259.

23. Lahl V, Sadler B, Schierenberg E: Egg development in parthenogenetic nematodes: variations in meiosis and axis formation. Int J Dev Biol 2006, 50(4):393-398.

24. Goldstein P, Wharton D: The synaptonemal complexes of the meiotic parthenogenetic Antarctic nematode Panagrolaimus davidi: karyotype analysis and three-dimensional reconstruction of pachytene nuclei. Cytobios 1996, 85(34I):8I-90.

25. Smith T, Wharton DA, Marshall C): Cold tolerance of an Antarctic nematode that survives intracellular freezing: comparisons with other nematode species. J Comp Physiol [B] 2008, I78(I):93-100.

26. Wharton DA, Barrett J, Goodall G, Marshall CJ, Ramlov H: Iceactive proteins from the Antarctic nematode Panagrolaimus davidi. Cryobiology 2005, 5 I (2): 198-207.

27. Wharton DA, Downes MF, Goodall G, Marshall CJ: Freezing and cryoprotective dehydration in an Antarctic nematode (Panagrolaimus davidi) visualised using a freeze substitution technique. Cryobiology 2005, 50(I):2I-28.

28. Wharton DA: The environmental physiology of Antarctic terrestrial nematodes: a review. J Comp Physiol [B] 2003, I73(8):621-628.

29. Wharton DA, Goodall G, Marshall CJ: Freezing survival and cryoprotective dehydration as cold tolerance mechanisms in the Antarctic nematode Panagrolaimus davidi. J Exp Biol 2003, 206(Pt 2):2I5-22I.

30. Wharton DA, Judge KF, Worland MR: Cold acclimation and cryoprotectants in a freeze-tolerant Antarctic nematode, Panagrolaimus davidi. J Comp Physiol [B] 2000, I70(4):32I-327.

31. Ramlov $\mathrm{H}$, Wharton DA, Wilson PW: Recrystallization in a freezing tolerant Antarctic nematode, Panagrolaimus davidi, and an alpine weta, Hemideina maori (Orthoptera; Stenopelmatidae). Cryobiology 1996, 33(6):607-613.

32. Wharton DA, To NB: Osmotic stress effects on the freezing tolerance of the antarctic nematode Panagrolaimus davidi. $J$ Comp Physiol [B] 1996, I66(5):344-349.

33. Wharton DA: Freeze-substitution techniques for preparing nematodes for scanning electron microscopy. J Microsc 1991, 164(Pt 3): 187-196.

34. Aroian R, Carta L, Kaloshian I, Sternberg PW: A free-living Panagrolaimus sp. from Armenia can survive anhydrobiosis for 8.7 years. J Nematol 1993, 25:500-502.

35. Shannon AJ, Browne JA, Boyd J, Fitzpatrick DA, Burnell AM: The anhydrobiotic potential and molecular phylogenetics of species and strains of Panagrolaimus (Nematoda, Panagrolaimidae). J Exp Biol 2005, 208(Pt I 2):2433-2445.

36. Cutter AD: Divergence times in Caenorhabditis and Drosophila inferred from direct estimates of the neutral mutation rate. Mol Biol Evol 2008, 25(4):778-786.

37. Fabian T], Johnson TE: Production of age-synchronous mass cultures of Caenorhabditis elegans. J Gerontol 1994, 49(4):B। $45-156$.

38. Shannon AJ, Tyson T, Dix I, Boyd J, Burnell AM: Systemic RNAi mediated gene silencing in the anhydrobiotic nematode Panagrolaimus superbus. BMC Mol Biol 2008, 9:58.

39. Wharton D: Comparison of the Biology and Freezing Tolerance of Panagrolaimus davidi, an Antarctic Nematode, From Field Samples and Cultures. Nematologica 1998, 44:643-653.

40. Tamura K, Dudley J, Nei M, Kumar S: MEGA4: Molecular Evolutionary Genetics Analysis (MEGA) software version 4.0. Mol Biol Evol 2007, 24(8): 1596-1599.

4I. Nadler SA, Carreno RA, Adams BJ, Kinde H, Baldwin JG, MundoOcampo M: Molecular phylogenetics and diagnosis of soil and clinical isolates of Halicephalobus gingivalis (Nematoda: Cephalobina: Panagrolaimoidea), an opportunistic pathogen of horses. Int I Parasitol 2003, 33( I0): I I I 5-1 I 25.

42. Nadler SA, De Ley P, Mundo-Ocampo M, Smythe AB, Patricia Stock S, Bumbarger D, Adams BJ, De Ley IT, Holovachov O, Baldwin JG: Phylogeny of Cephalobina (Nematoda): molecular evidence 
for recurrent evolution of probolae and incongruence with traditional classifications. Mol Phylogenet Evol 2006, 40(3):696-7II.

43. Denver DR, Morris K, Thomas WK: Phylogenetics in Caenorhabditis elegans: an analysis of divergence and outcrossing. Mol Biol Evol 2003, 20(3):393-400.

44. Kimura M: The Neutral Theory of Molecular Evolution. Cambridge University Press, Cambridge, UK; 1983.

45. Zuckerkandl E, Pauling L: Molecular Disease, Evolution, and Genetic Heterogeneity. In Horizons in Biochemistry New York, NY: New York: Academic Press; 1962:189-225.

46. Rozas J, Sanchez-DelBarrio JC, Messeguer X, Rozas R: DnaSP, DNA polymorphism analyses by the coalescent and other methods. Bioinformatics 2003, I 9(1 8):2496-2497.

47. Hong RL, Svatos A, Herrmann M, Sommer RJ: Species-specific recognition of beetle cues by the nematode Pristionchus maupasi. Evol Dev 2008, I0(3):273-279.

48. Kiontke K, Sudhaus W: Ecology of Caenorhabditis species. WormBook 2006: I-I4.

49. Cardoza YJ, Moser JC, Klepzig KD, Raffa KF: Multipartite symbioses among fungi, mites, nematodes, and the spruce beetle, Dendroctonus rufipennis. Environ Entomol 2008, 37(4):956-963.

50. Liau WS, Gonzalez-Serricchio AS, Deshommes C, Chin K, LaMunyon $\mathrm{CW}$ : A persistent mitochondrial deletion reduces fitness and sperm performance in heteroplasmic populations of $C$. elegans. BMC Genet 2007, 8:8.

5I. Floyd R, Abebe E, Papert A, Blaxter M: Molecular barcodes for soil nematode identification. Mol Ecol 2002, I I(4):839-850.

52. Hu M, Chilton NB, Gasser RB: Long PCR-based amplification of the entire mitochondrial genome from single parasitic nematodes. Mol Cell Probes 2002, 16(4):26I-267.

53. Elkin CJ, Richardson PM, Fourcade HM, Hammon NM, Pollard MJ, Predki PF, Glavina T, Hawkins TL: High-throughput plasmid purification for capillary sequencing. Genome Res 200I, II(7):1269-I274.

Publish with Bio Med Central and every scientist can read your work free of charge

"BioMed Central will be the most significant development for disseminating the results of biomedical research in our lifetime. "

Sir Paul Nurse, Cancer Research UK

Your research papers will be:

- available free of charge to the entire biomedical community

- peer reviewed and published immediately upon acceptance

- cited in PubMed and archived on PubMed Central

- yours - you keep the copyright 